\title{
Test Suites as a Source of Training Data for Static Analysis Alert Classifiers
}

\author{
$1^{\text {st }}$ Lori Flynn \\ Software Engineering Institute \\ Carnegie Mellon University \\ Pittsburgh, USA \\ lflynn@sei.cmu.edu
}

\author{
$2^{\text {nd }}$ William Snavely \\ (former) Software Engineering Institute \\ Carnegie Mellon University \\ Pittsburgh, USA \\ will.snavely@gmail.com
}

\author{
$3^{\text {rd }}$ Zachary Kurtz \\ (former) Software Engineering Institute \\ Carnegie Mellon University \\ Pittsburgh, USA \\ zkurtz@pm.me
}

\begin{abstract}
Flaw-finding static analysis tools typically generate large volumes of code flaw alerts including many false positives. To save on human effort to triage these alerts, a significant body of work attempts to use machine learning to classify and prioritize alerts. Identifying a useful set of training data, however, remains a fundamental challenge in developing such classifiers in many contexts. We propose using static analysis test suites (i.e., repositories of "benchmark" programs that are purposebuilt to test coverage and precision of static analysis tools) as a novel source of training data. In a case study, we generated a large quantity of alerts by executing various static analyzers on the Juliet $\mathrm{C} / \mathrm{C}++$ test suite, and we automatically derived ground truth labels for these alerts by referencing the Juliet test suite metadata. Finally, we used this data to train classifiers to predict whether an alert is a false positive. Our classifiers obtained high precision $(\mathbf{9 0 . 2 \%})$ and recall $(\mathbf{8 8 . 2 \%})$ for a large number of code flaw types on a hold-out test set. This preliminary result suggests that pre-training classifiers on test suite data could help to jumpstart static analysis alert classification in data-limited contexts.
\end{abstract}

Index Terms-static, analysis, alert, classification, rapid, precise, test suite, Juliet

\section{INTRODUCTION}

Flaw-finding static analysis (FFSA) tools typically generate large volumes of code flaw alerts including many false positives. To save on human effort to triage these alerts, a significant body of work attempts to use machine learning to classify and prioritize alerts. Identifying a useful set of training data, however, remains a fundamental challenge in developing such classifiers in many contexts. We propose using static analysis test suites (i.e., repositories of "benchmark" programs that are purpose-built to test FFSA tools) as a novel source of training data for a wide range of "conditions" (types of code flaws). In a case study, we generated a large quantity of alerts by executing various FFSA tools on the Juliet test suite, and we automatically derived ground truth labels for these alerts by referencing the Juliet test suite metadata. Finally, we used this data to train classifiers to predict whether an alert is a false positive, and tested the classifiers on hold-out data.

This paper focuses on warnings from FFSA tools that look for security flaws. A checkerID is a unique string or regular expression in a tool's alerts for that flaw type. We use "coding taxonomy" to mean a named set of coding rules, weaknesses, standards, or guidelines (e.g., CERT coding rule [13] or
Common Weakness Enumeration (CWE [21])). Each rule or weakness is considered a single condition. An FFSA tool's alert may be mapped to conditions in one or more coding taxonomies (e.g., [22] lists many FFSA tools that provide CWE output). "Alert fusion" refers to the practice of unifying alert information from different tools which map to the same condition in the same part of the code (e.g., same line of same file). Fusion may be imprecise, e.g., a particular CWE may occur in two different parts of the same line of code. 1

\begin{tabular}{|c|c|}
\hline Term & Definition \\
\hline SA tool & $\begin{array}{l}\text { static analysis tool that analyzes code without } \\
\text { running it }\end{array}$ \\
\hline FFSA tool & flaw-finding static analysis tool \\
\hline SA alert & $\begin{array}{l}\text { static analysis alert (warning) about a particu- } \\
\text { lar type of flaw }\end{array}$ \\
\hline Checker & $\begin{array}{l}\text { analysis for a particular type of code flaw, by } \\
\text { a particular FFSA tool }\end{array}$ \\
\hline CheckerID & checker name \\
\hline Test suites & $\begin{array}{l}\text { repositories of "benchmark" programs that are } \\
\text { purpose-built to test FFSA tools }\end{array}$ \\
\hline Condition & $\begin{array}{l}\text { a constraint or property of validity with which } \\
\text { code should comply. FFSA tools try to detect } \\
\text { if code violates conditions. }\end{array}$ \\
\hline Alert fusion & $\begin{array}{l}\text { unifying alert information from different tools } \\
\text { which map to the same condition and code } \\
\text { location }\end{array}$ \\
\hline Coding taxonomy & $\begin{array}{l}\text { a named set of coding rules, weaknesses, stan- } \\
\text { dards, or guidelines }\end{array}$ \\
\hline
\end{tabular}

Table I summarizes key terminology used in this paper.

\section{A. Related Work}

Our work uses the Juliet $\mathrm{C} / \mathrm{C}++\mathrm{v} 1.2$ test suite [12]. The Juliet test suites [12] provide example test programs with

${ }^{1}$ AST 2021, May 20-21, 2021. This is the authors' version of the work. This material is based upon work funded and supported by the Department of Defense under Contract No. FA8702-15-D-0002 with Carnegie Mellon University for the operation of the Software Engineering Institute, a federally funded research and development center. References herein to any specific commercial product, process, or service by trade name, trade mark, manufacturer, or otherwise, does not necessarily constitute or imply its endorsement, recommendation, or favoring by Carnegie Mellon University or its Software Engineering Institute. Carnegie Mellon ${ }^{\circledR}$ and CERT ${ }^{\circledR}$ are registered in the U.S. Patent and Trademark Office by Carnegie Mellon University. DM180144 
multiple subtypes of each CWE addressed (e.g., separate variant programs instantiating the flaw using an integer or a string), and for most of those subtypes also provides variant test programs involving variants of control, data, and/or type flow (e.g., a simple example with the flaw completely within a function, versus an example with control and data flow through multiple function calls and involving data pointers). Test suites are usually used to test and compare vulnerability analysis tools [10], [20] or test system defenses [5], but our work uses test suites in a new way: to rapidly build labeled data for SA alert classifier development.

NIST provides over 600,000 cost-free, open-source test suite programs (including the Juliet test suite) in its Software Reference Dataset (SARD) [24], along with metadata identifying each test's known flaw conditions and the flaw locations. Our method could be applied even more broadly with additional test suites including the others hosted by NIST.

Delaitre et al. tested security flaw-finding SA tools on the Juliet test suite (their research mostly focused on the Java tests) and on average those tools found about $20 \%$ of weaknesses in basic test cases (no added complexity). They found that complex control flow or data flow constructs significantly reduced the tools' success rates, and identify flaws with highest and fewest findings across a set of anonymized FFSA tools [9]. Since single FFSA tools have different coverage of code flaws (warning about some but not others) [6], [9], multiple FFSA tools may be used to find more code flaws [25]. This approach, however, compounds the problem of generating too many alerts to deal with, including too many false positives. Our work uses multiple FFSA tools and addresses the issue of handling the alerts. Our work builds on the findings in [9] by gathering data on FFSA tool performance for variant flows of control, type, and data for CWE sub-types and then using that for classifier development.

Pugh and Ayewah found a mean time of 117 seconds per SA alert review, from analyzing data from 282 Google engineers that made over 10,000 manual SA alert determinations [3]. One example of insufficient labeled data as a barrier comes from our previous work with 3 large organizations that do software development, where lack of data covering more types of flaws resulted in classifier incorporation being impractical for them [2]. Even when an organization has large audit archives, if the auditors have not used a consistent set of audit rules and a well-defined auditing lexicon, the data may not be useful (and most organizations don't have a well-defined auditing lexicon and auditing rules) [28]. Data-rich Google developed $85 \%$ accurate classifier models predicting FindBugs false positives [27]. Cross-project classifier prediction is an area of research developed to address insufficient labeled data for a code project. Our work is intended to rapidly develop a large quantity of labeled data archives using labeled alerts on test suite, creating classifiers to do cross-project defect prediction on production code alerts. Nam and Kim [23] discuss issues related to cross-project defect prediction: some features in different projects may correlate to predictions in different ways, but the rationale for doing cross-project prediction is a hypothesis that some features are helpful for developing classifiers that work well across projects. They use the magnitude of metric values to do cross-project defect prediction. Their work focuses on determining a subset of labeled data and features within that data to use, while our work focuses on development of a labeled dataset covering many conditions that develops precise classifiers. Zhang et al. [32] did crossproject prediction, showing improvement of classification results using a connectivity-based unsupervised classifier. A connectivity-based unsupervised classifier could possibly be used on data from the labeled audit archives produced from test suites and alerts from production code. Jing et al. [16] does cross-company defect prediction including cross-project prediction, using features that include the name of company that developed code for the line/function/file/class/program related to the alert. A labeled dataset from our system could be used in conjunction with their techniques.

Bessey et al. [6] found many issues with using SA in practice, including tools ignoring constructs and thus producing many false positive alerts, users wrongly labeling diagnostics they find confusing as false, and user difficulty dealing with many alerts resulting in tools producing less (possibly-true) alerts about possible defects than they could. Beller et al. found that, in practice, few open-source projects have FFSA tools integrated closely with their workflows, and most of those projects do not mandate that a codebase should be warningfree [4]. The goal of our work is to make it easier to use FFSA tools to effectively find and fix prioritized defects, including for open-source projects.

Heckman and Williams [14] did an extensive survey of methods that classify and prioritize actionable alerts, detailing 21 peer-reviewed studies. Our method uses 5 of the approaches (alert type selection, contextual information, data fusion, machine learning, and mathematical and statistical models) discussed in the paper, and doesn't use the other 3 (dynamic detection, graph theory, and model checking). Our method uses 2 of the artifact characteristics categories (alert characteristics, code characteristics), and doesn't use the other 3 (source code repository metrics, bug database metrics, and dynamic analysis metrics). No previous work in their survey (nor in any research publication we have found since then) involves using test suites to automatically label data to create classifiers.

The approach by Kremenek et al. [18] does not merge sets of alerts from different tools, so alerts from a tool are prioritized in a set according to that tool's ranking rather than individually. Alerts are ranked by correlating data per FFSA tool, using features from tools and from codebases [19]. Sets of alerts from different tools are ordered relatively (all alerts from one tool are prioritized below all alerts from another tool, never interspersed), with no alert fusion. The labeled data archives developed in our work could be used in combination with labeled production alerts in a similar adaptive heuristic.

Our own earlier research [1], [2] developed classifiers in many ways similar to the current work: labeled audit archives were used, with fused alerts from different tools that map to 
the same condition, line number, and filepath. However, that work was only able to develop accurate classifiers for 3 CERT $\mathrm{C}$ coding rules with single rule data, despite using a significant quantity of audit archives. Those audit archives include data from 8 years of CERT analysis on 26 codebases, plus new audit data provided by three collaborating organizations over the course of a year where the collaborators audited SA alerts for their own codebases using an auditing lexicon and auditing rules we developed [28]. Our current work addresses that labeled-data quantity problem.

\section{New Method for Rapidly Generating LAbeled ALERT ARCHIVES}

To quickly generate a large quantity of labeled data for classifier development, we developed a software system that uses test suites, as shown in Fig. 1. Data for classifier development uses results for each FFSA tool on variant flows of control, type, and data for CWE sub-types. Resultant classifier development potentially can use this information about individual FFSA tool precision (percent correct or true positive) in combination with type of flow complexity, CWE sub-type, and the set of FFSA tools that alert to more precisely predict if a given alert is true or false.

We ran 8 FFSA tools on the 61,387 tests (covering 118 CWEs) in the Juliet Test Suite for C/C++ v1.2 [12], including popular proprietary tools and open-source tools. We developed scripts that parse the FFSA tool output and upload alert data to a database, including the checkerID plus the filepath and line number of the possible defect. Due to licensing restrictions, we cannot name proprietary tools we used nor their performance. The open-source FFSA tools we used are Cppcheck [30], Rosecheckers [29], and GCC [26]. GCC is a compiler, but like all compilers it statically analyzes the code, plus it outputs warnings (when run with parameters such as these: - Wall - Wextra - Wpointer - arith - Wstrict prototypes - Wformat - security) that can be mapped (as regular expressions) to code flaw taxonomies [8].

We generated additional features for the classifiers to train on by running three code metrics tools on the test suite: (Lizard [31], CCSM [7], and a proprietary tool (anonymous)). All the tools provide counts of significant lines of code, complexity, and cohesion. Lizard provided the fewest metrics and CCSM provided the most. Our database correlates each alert with applicable code metrics.

\section{A. Deriving Ground Truth Alert Verdicts}

The Juliet Test Suite contains two kinds of metadata that are relevant for determining the validity of alerts:

- a manifest file: This is an XML file that provides precise flaw information including line number, CWE, and filepath.

- function names: Documentation for the test suite says that if the function name includes the string GOOD then the particular CWE does not occur in it, but if it includes the string BAD then the CWE does occur in the function. We gathered information about filepath and line numbers covered by each function name that contains GOOD or $\mathrm{BAD}$, as well as the CWE indicated (usually by filename).

Note that both the manifest file and the function names provide only CWE-specific flaw information. In general, a line of code marked BAD for one code flaw type could be flawless with respect to all other code flaw types. Thus, we can use the metadata flaw information to determine the validity of an alert only when we can establish that the alert's checkerID is for a flaw of the same type as a flaw referenced in the metadata. The test suite metadata does not identify every CWE weakness in the Juliet code nor all locations of the CWE weaknesses, so an alert that doesn't map to the test suite metadata cannot be automatically labeled using the metadata. In other words, if an alert's CWE doesn't match the test suite metadata's CWE, the metadata can't be used to label the alert true or false.

Publicly-available mappings between checkerIDs and CWEs are available for many of the FFSA tools that we tested. We fused alerts from this set of tools, producing a set of fused alerts with known CWE designations. We then determined verdicts (i.e. classifier ground truth labels) for each fused alert as follows:

- If the manifest includes a record of a flaw for the same filepath, line number, and CWE as the alert, then set verdict $=$ True, indicating that the alert is a true positive.

- If the defect alert matches any line within a function name with GOOD and the alert's CWE matches the CWE associated with the function, then set verdict $=\mathrm{False}$, indicating a that the alert is a false positive.

Applying the above procedure resulted in 36,968 TP fused alerts, 84,269 FP fused alerts, and fused alerts with no determination. Note that the procedure defined above conservatively refrains from assigning a verdict for an alert when (a) the alert falls within a function labeled BAD but (b) the metadata flaw line number does not match the line number of the alert.

Automatically generating this volume of alert verdicts is potentially a nontrivial cost savings. As per Section I-A, manually auditing alerts typically takes on the order of 117 seconds per alert. Manually generating our 121,237 alert verdicts at this rate might have taken 3,940 hours. However, manual auditing and natural code would likely not cover many of the conditions in the Juliet test suite, since many conditions rarely exist in natural code. Even if FFSA tools were run on a large number of natural codebases (which would take a lot of time and computation) and alerts were found for many conditions, it might take many manual audits before both true and false determinations could be made. Realistically, it would take an enormous amount of manual auditing time (and money to pay for it), to develop that much data.

In comparison, incorporating a new FFSA tool into our system takes approximately 24 hours of developer effort, to code a parser for the FFSA tool output (unless it uses the new SARIF standard output format /citesarif) and otherwise integrate into the system. After the FFSA tool is run on the test suite, the script the developer wrote is run on the output, 


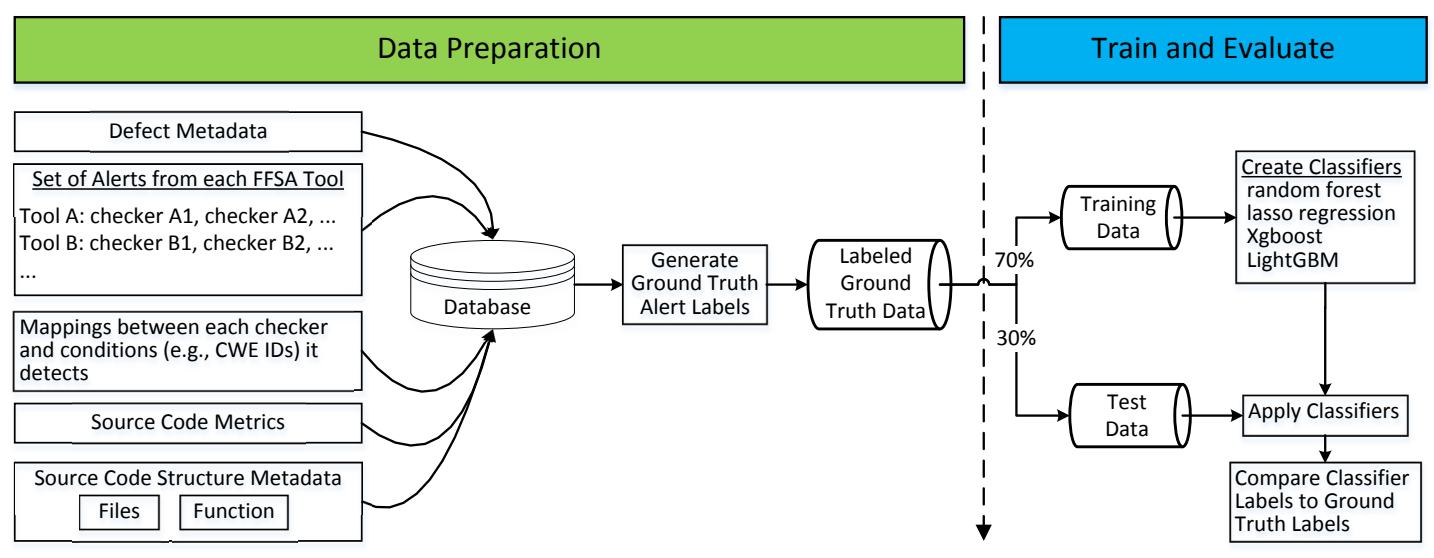

Fig. 1. System for automated alert labeling and classifier training

and then labeling the alerts automatically and rapidly happens. Adding a new test suite to our system similarly involves using standard interfaces to adapt the system to integrate another test suite. However, adding a new test suite takes more manual effort because each test suite has new unique compile and test requirements, and for non-SARD test suites the metadata format must be adapted to the SARD metadata format. Considering the effort required to add more FFSA tools and test suites, the new automated system clearly scales to generate much more labeled data at much lower cost than manual analysis.

\section{B. Speculative Mapping Method}

For FFSA tools that did not have publicly-available checker mappings to CWEs, we developed a speculative mapping method to estimate these mappings. Since FFSA tools often find different sets of defects, we hoped to obtain greater CWE coverage without the cost of manual mapping between the FFSA tools' checkers and CWEs.

Our speculative mapping method treats a co-occurrence of a test suite metadata CWE designation for a line of code and an alert on that same line of code as evidence that the alert's checkerID was designed to detect the kind of flaw indicated by that CWE. Such a co-occurence is a checker-CWE match.

We summarize this evidence by counting all checker-CWE matches. That is, for every checker-CWE pair, we count how many times an alert issued by that checker falls on the same line as a code flaw of that CWE. A checker may match multiple CWEs, however, so it's not always obvious which CWE a checker is the best match for.

We define two checker-CWE match rate percentages to guide the speculative mapping of checkerIDs to CWEs. Let $m_{i j}$ denote the count of checker-CWE matches between checker $i$ and CWE $j$; let $m_{i}=\sum_{j} m_{i j}$, the total count of all checker-CWE matches involving checker $i$; and let $m_{j}=\sum_{i} m_{i j}$, the total count of all checker-CWE matches (for the tool being speculatively mapped) involving CWE $j$.
Finally, we define the

$$
\text { forward match percentage }=100 \frac{m_{i j}}{m_{i}}
$$

and the

$$
\text { backward match percentage }=100 \frac{m_{i j}}{m_{j}} .
$$

Conceptually, these match rate percentages are alternative ways to measure the relative frequencies by which alerts from each checker fall on the lines of particular conditions (CWEs) that are recorded in the test suite manifest.

For each checker, we establish a preliminary assignment of that checker to the CWE that has the greatest forward (backward) match rate percentage with that checker. Finally, we fix a threshold greater than 0 . All preliminary assignments for which the forward (backward) match rate exceeds the threshold become speculative mappings. (Section III-D details the specific thresholds used.)

Proceeding with the assumption that the speculative mappings are correct allows us to increase the number of alerts with ground truth verdicts. Every alert from a speculativelymapped checker has an associated CWE (via the speculative mapping), and we assign verdicts for such alerts just as described in Section II-A

1) Errors in Speculative Mappings: Some speculative mappings are wrong. For example, the unmapped tool's checker may be alerting about a type of code flaw on that code line that the test suite manifest does not mention. Other mappings are only partially correct. That is the case when a CWE and a tool checker do not have an EQUALS relationship, but instead have a SUBSETOF, SUPERSETOF, or PARTIALLY OVERLAPPING relationship. Some mappings will be missed by speculative mapping. The unmapped tool might detect the code flaw specified in the test suite manifest, but assign the flaw location to code location $X$ while the manifest assigns it to code location $Y$. Fault and failure location may differ. For example, if a failure is caused by an out of bound array index, the tool and manifest might locate 
the fault differently. One could define it in the very line of the access or in a function that did not sanitize before that.

2) Test Mappings Rationale and Alternatives: We tested forward and backward speculative matching with varying thresholds, to gather empirical data related to checker to CWE mappings. We tried to identify generally-useful thresholds or mapping directions. If the actual relationship is $E Q U A L S$ then we would expect close to a $100 \%$ match. If the actual relationship is SUBSETOF or SUPERSETOF then we would expect the forward and backward speculative matching results to be different. If the relationship is PARTIALLY OVERLAPPING the forward and backward speculative matching results could be similar or different. Alternative formulas not tested in this work might produce useful results, such as: combined_match_percentage $=0.5 *$ forward $+0.5 *$ backward.

\section{Automated Speculative Mapping with Manual Verification}

We identified an alternative to using the automated speculative mappings alone. In this alternative, we start with speculative mappings and then perform manual verification of the mapping. There are approximately $700 \mathrm{CWE}$ and static analysis tools may have hundreds of checker IDs. Validating 70,000 possible checker mappings (in this example, for a tool with only 100 checker IDs) would be very time-consuming, as it requires a precise understanding of both the checker definition (which takes on average time (D_checker)) and the CWE definition (which takes on average time $\left(D_{-} C W E\right)$ ), and after that determination of the relationship between checker and CWE (which takes on average time(Relationship)) for a total time in our example of $70,000 * \operatorname{time}\left(D_{-} C W E\right) *$ time $\left(D \_\right.$checker $) *$ time $($ Relationship $)$. However, speculative mapping reduces the candidate mappings to a far smaller number, with each candidate mapping by definition having evidence supporting the possible mapping. Three different speculative CWE mappings per each tool checker ID is far more than we actually observed. Using that overestimate, for our example the amount of time required to do such mapping would be $300 *$ time $\left(D_{-} C W E\right) *$ time $\left(D \_\right.$checker $) *$ time(Relationship), which is a factor of 233 less than 70,000.

\section{BUILDING AND TESTING CLASSIFIERS}

The classifier task is to determine whether a fused alert is a True Positive (TP) or a False Positive (FP) on the basis of various code metrics and other features associated with each alert. Section III-A explains how we divided the labeled alert archives into a training set and a test set. Section III-B introduces the classifiers. Section III-C describes the performance of the classifiers for fused alerts excluding all speculatively mapped alerts and analyzes the importance of specific features. Section III-D shows how overall performance differs after including speculatively mapped alerts in the training data.

\section{A. Training data versus test data}

We defined a non-speculative training data set and numerous speculative training data sets based on the inclusion or exclusion of alerts with speculatively-mapped checkerIDs. The hold-out test set, by contrast, involved only alerts with non-speculative mappings to ensure a consistent comparison between a classifier that is alternatively trained on the two training sets.

To be precise about the test train splits, define the following sets of raw (pre-fused) alerts and fused alerts:

- $A_{\text {mapped }}$ : the raw alerts with checkerIDs that have known CWE mappings.

- $A(T, d)$ : the raw alerts in $A_{\text {mapped }}$ together with the alerts whose checkerID that is speculatively mapped to a CWE above the threshold $\mathrm{T}$ on the match percentage in direction $d \in\{$ forward, backward $\}$, as per Section II-C.

- $A_{\text {pure }}$ : the raw alerts that do not share a line with any other alert that has a speculatively-mapped checkerID (for every threshold and match percentage direction).

- $A F_{\text {mapped }}$ : the fused alerts derived from $A_{\text {mapped }}$.

- $A F(T, d)$ : the fused alerts derived from $A(T, d)$.

- $A F_{\text {pure }}$ : the fused alerts derived from $A_{\text {pure }}$.

- AF test: a stratified (on verdict and CWE) random sample of the fused alerts in $A F_{\text {pure }}$. This represents a "pure" test set not intertwined with speculative mappings.

- $A F_{n o n-s p e c u l a t i v e}$ : the fused alerts in $A F_{\text {mapped }}$ excluding those in $A F_{\text {test }}$.

- $A F_{\text {speculative }}(T, d)$ : the fused alerts in $A F(T, d)$, excluding those in $A F_{\text {test }}$.

To summarize, we started by defining our single test set $A F_{\text {test }}$ which included 36,445 fused alerts, or about $30 \%$ of the fused alerts excluding speculative mappings. We then defined the training set $A F_{n o n-s p e c u l a t i v e ~}$ to include all the remaining fused alerts, excluding speculative mappings. Finally, we defined a series of training sets $A F(T, d)$ that include all of the alerts in $A F_{n o n-s p e c u l a t i v e ~}$ but also include varying subsets of the alerts with speculatively-mapped checkerIDs.

Table II shows the number of fused alerts in the training set $A F_{\text {speculative }}(T, d)$ for each combination of threshold $T \in\{0 \%, 5 \%, 25 \%, 50 \%, 75 \%, 100 \%\}$ and match direction $d$. At the most permissive setting (with a threshold of 0 ), the number of fused alerts in the training data is 464,887 , approximately twice the 121,237 fused alerts in the main training set.

\section{B. Classifiers and performance metrics}

We used the $\mathrm{R}$ statistical programming language to run four different classifiers: LightGBM, XGBoost, the H2O . a i implementation of random forests, and the $\mathrm{g}$ lmnet implementation of lasso-regularized logistic regression. All of these except for the lasso regression are based on decision trees. LightGBM and XGBoost won acclaim on Kaggle [17] and are both examples of regularized Gradient Boosting Machines. Random forest and lasso regression are both generic algorithms; we used the the random forest implementation from $\mathrm{h} 2 \mathrm{O} . \mathrm{ai}$ and the lasso implementation in the glmnet package.

Our purpose for trying several classifiers is to identify at least one one that generates highly accurate predictions on a hold-out test set. In particular, inference regarding the 


\begin{tabular}{lrrrrrr}
\hline Match direction & $0 \%$ & $5 \%$ & $25 \%$ & $50 \%$ & $75 \%$ & $100 \%$ \\
\hline backward & 464887 & 308038 & 169370 & 149926 & 137128 & 134374 \\
forward & 464887 & 426927 & 296259 & 222108 & 193622 & 134374 \\
\hline
\end{tabular}

TABLE II

NUMBER OF FUSED ALERTS IN THE TRAINING DATA FOR EACH SPECULATIVE MAPPINGS THRESHOLD

importance of individual features (including multicollinearityinduced variance in feature effects) was not a primary focus. Although we did some experimentation to choose reasonable hyperparameters for each classifier, we largely accepted default settings, treating each algorithm as a black-box. All four of these algorithms are sophisticated in the sense that they tend to perform well on nearly arbitrary sets of features.

For each of these classifiers, a prediction for an alerts is a number between 0 and 1 that represents an estimated probability that the alert has verdict $=$ True. We round these predictions to generate binary classification output as needed to compute metrics like precision and recall.

Our primary metric for classifier performance is the area under the receiver operating characteristic curve (AUROC). The AUROC is useful for comparing the overall performance of classifiers when the classifier output is probabilistic. The AUROC gives partial credit to probabilistic predictions: When the true label is 1 , a prediction of 0.4 scores much better than a prediction of 0 , for example. The AUROC penalizes false positive probability mass and false negative probability mass equally.

In addition to the AUROC, we compute the precision, recall, and accuracy, since these standard metrics are relatively easy to interpret. The precision is the fraction of predicted TP alerts that were indeed TP, and the recall is the fraction of TP alerts that were successfully predicted to be TP. Note that precision is meaningless when the classifier does not classify any issues as TP, and so precision values for certain subsets of the test set are missing. Similarly, recall is meaningless when the labeled alerts contain no TP alerts, as there is nothing to be "recalled".

\section{Results without speculative mappings}

This section summarizes performance of the classifiers on the test set $A F_{\text {test }}$ after training on the main training data $A F_{\text {non-speculative }}$ (as per Section III-A.

Table III summarizes the average performance of the different classifiers over the entire test set. Table IV summarizes the performance of LightGBM, our best classifier, on all CWE IDs for which at least one test data point was available. Note that that test count is the number of fused alerts available for testing for each CWE ID, and TP rate is the fraction of the testing alerts that were TP.

Table $\mathrm{V}$ shows the same performance statistics on groups of alerts associated with selected CERT rules. For each CERT secure coding rule, we identified the set of relevant CWE test programs based on previously-established mappings between the taxonomies, documentation about the test suite, and manual inspection of the test suite programs. We subsequently identified the set of fused alerts applicable to each CERT rule via their CWE designations. These sets are not mutually disjoint in general; the CWE of each fused alert can correspond to no CERT rule, one CERT rule, or multiple CERT rules. Thus, the values of test count need not correspond to the counts in Table IV.

A cursory examination of accuracy in Tables IV and $\mathrm{V}$ suggests that the classifier is often accurate across many different kinds of rules. Some of these results stand out. The first CWE (457) and second CERT Rule (ARR30-C) both have very low TP rates; such low TP rates are the source of a great deal of wasted triaging effort. Our classifier, however, performed at nearly $100 \%$ accuracy on these sets of alerts, correctly classifying them as FP.

There are limitations, however, to how broadly we can interpret this success. Juliet Test Suite is not a representative sample of code in general. The kinds of code artifacts that trigger alerts from various checkers on the test suite could differ significantly from other code bases, degrading the performance of our classifier outside of the test suite.

\begin{tabular}{lrrrr}
\hline Classifier & Accuracy & Precision & Recall & AUPRC \\
\hline rf & 0.947 & 0.898 & 0.902 & 0.979 \\
lasso & 0.880 & 0.891 & 0.628 & 0.833 \\
xgboost & 0.949 & 0.922 & 0.882 & 0.974 \\
lightgbm & 0.958 & 0.917 & 0.928 & 0.985 \\
\hline \multicolumn{4}{c}{ TABLE III }
\end{tabular}

AVERAGE PERFORMANCE OF DIFFERENT CLASSIFIERS

We calculated the top 10 features in terms of the total gain of each feature's splits in the underlying LightGBM trees, shown in Table VI (metrics from a proprietary tool anonymized). This importance ranking is not guaranteed to identify all significant features, and the precise ordering of features is not very meaningful (in the presence of multicollinearity, for example). Such a ranking, however, is useful for identifying at least a sample of the moderate-to-highly-significant features. We use this to provide an example of the patterns that the classifier detects.

The feature with the highest information gain is the field FUNC_CALLED_BY_LOCAL, from the CCSM metrics tool, representing the "number of local functions calling this function". Fig. 3 shows that alerts are almost always true positives when the function that contains the alerted line of code is called by at least one local function.

\section{Results using speculatively mapped alerts}

We re-trained LightGBM - our best classifier - separately on each of the training data sets $A F_{\text {speculative }}(T, d)$ (as per Sections II-C, III-A. Experimenting with setting various thresholds $T$ on the $d=$ forward (or backward) match percentages is a way to include speculatively mapped alerts of 


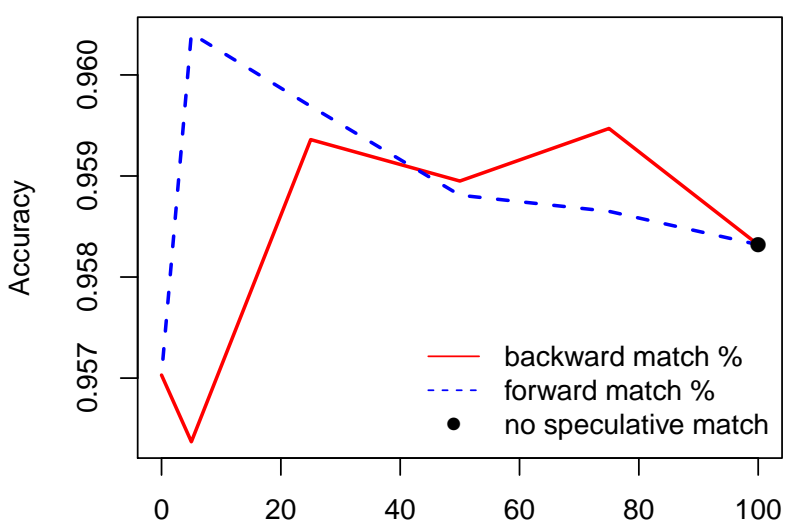

Match percentage threshold (lower is more inclusive)

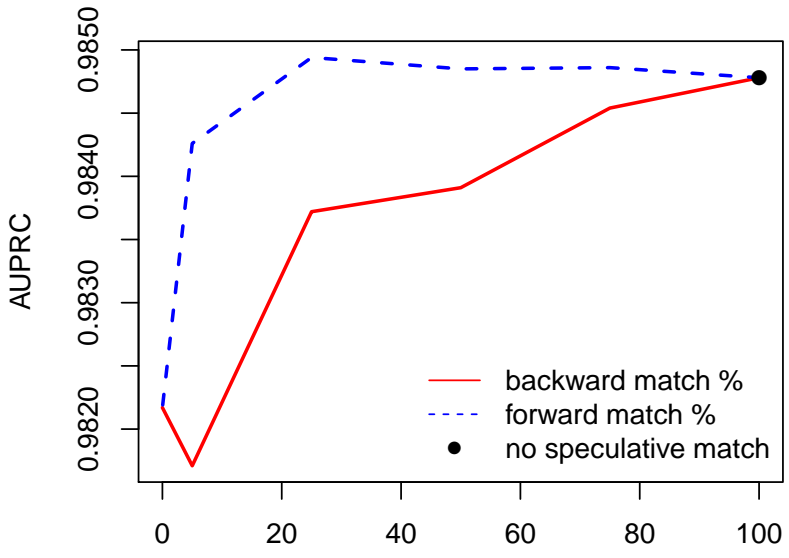

Match percentage threshold (lower is more inclusive)

Fig. 2. Assessing the value of training a classifier on speculatively mapped alerts

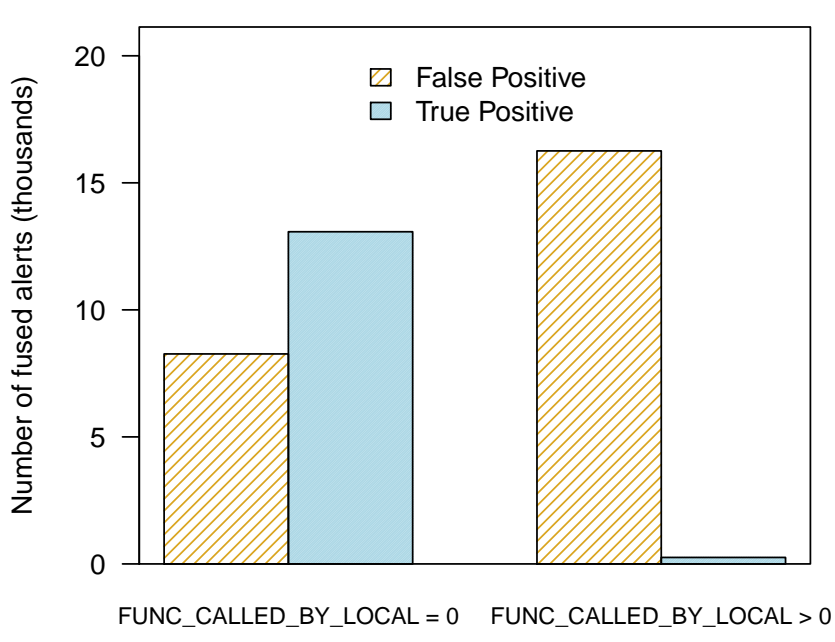

Fig. 3. Labeled alerts vs. function caller counts

varying levels of "speculativeness" in the training data for the classifiers. This section summarizes these alternative training data sets in terms of the performance of the resulting trained classifier on test set $A F_{\text {test }}$.

Figure 2 shows performance metrics for LightGBM (our best classifier) at each matching threshold. The red (blue dashed) lines show the performance at each threshold applied to the backward (forward) match percentage. To understand whether inclusion of speculatively-mapped alerts in the training set improves the performance of the classifier, compare the red and blue lines against the black point at the right-hand side of each graph, which marks the performance of LightGBM trained on data that excludes all speculative mappings (i.e. a threshold just greater than 1).

From Figure 2, we conclude that the addition of the speculative alerts did not substantially affect the performance of the classifiers. This was surprising in two ways, since we had (1) expected performance to be slightly better with (as opposed to without) the inclusion of high-threshold speculatively-mapped alerts and (2) expected performance to degrade with the inclusion of alerts at the lowest threshold, since many of the speculative mappings are presumably incorrect and might "poison" the training data.

Looking past the overall average classifier performance to the performance on individual CWEs, there is a possibility that speculative mappings may improve the classifier for individual CWEs that are otherwise severely underrepresented in the training data. At the most inclusive speculative mappings configuration (threshold 0), there are 102 CWEs in the training data, significantly more than the 82 CWEs in the nonspeculative data.

We observed, however, that the classifier accuracy for well-represented CWEs was not significantly higher than the accuracy on underrepresented ones. For instance, the mean accuracy for the top 20 most-common CWEs in our training data was 0.946 , only slightly greater than the accuracy of 0.942 obtained for the 20 least common CWEs. This suggests that there is not a strong relationship between the number of fused alerts in the training data for CWEs of a particular type and the resulting classifier performance for that CWE. That is, it appears that signals that the classifier learns are not particularly CWE-specific, such that the classifier may do well even for new alerts corresponding to CWEs that were not represented in the training data.

\section{DATA}

We have published the open-source data used to develop classifiers and make mappings in this work, within the "RC_Data" dataset [11] That dataset includes Juliet Java test suite data that is not part of this paper's case study, but that data was developed using the auto-labeling system discussed in this paper.

\section{COnClusions, Limits, Future Work}

We developed a novel method that uses test suites to automatically generate a large quantity of labeled data for SA alert classifier development. We implemented this in a software 
system and then in a case study, we generated a large quantity of labeled data for many different conditions, using the Juliet test suite. Initial tests of the resulting classifiers on partitioned alerts from the test suite data show high accuracy for a large number of code flaw types. We developed an automated method to map checkerIDs to code flaw taxonomy conditions specified in test suites. We also created a mised automatedand-manual method that can greatly reduce mapping effort compared to fully-manual mapping while being more correct than fully-automated mappings.

With incorporation of test suites that cover more conditions (e.g., more CWEs), we expect that the methods demonstrated here would lead to successful classifiers for those additional conditions. In general, the method and software system developed can be used with any FFSA and code metrics tools to rapidly develop labeled data archives with standard additions to the system: Incorporating a new tool requires a parser and uploads to the database, while any new FFSA tool requires checkerID mappings. The general method can use additional artifact characteristics (source code repository metrics, bug database metrics, and dynamic analysis metrics), but the software system we developed would need to be extended.

The Juliet test suite programs are small (generally consisting of 1-3 short files) and are synthetically constructed. Our future work includes testing the classifiers on natural codebases, including both widely-used publicly-available cost-free opensource codebases and non-public proprietary codebases belonging to collaborators. Also, we have started to include test suites like STONESOUP [15] with much larger test programs (in the case of STONESOUP, widely-used natural test programs injected with flaws) that also have more complex control, data, and type flows than the Juliet test suite.

\section{REFERENCES}

[1] Prioritizing alerts from static analysis to find and fix code flaws. https://insights.sei.cmu.edu/sei_blog/2016/06/ prioritizing-alerts-from-static-analysis-to-find-and-fix-code-flaws.html 2016. Accessed: 2016-06-27.

[2] Prioritizing security alerts: A dod case study. https://insights.sei. cmu.edu/sei_blog/2017/01/prioritizing-security-alerts-a-dod-case-study. html January 2017.

[3] Nathaniel Ayewah and William Pugh. The google findbugs fixit. In Proceedings of the 19th international symposium on Software testing and analysis, pages 241-252. ACM, 2010.

[4] Moritz Beller, Radjino Bholanath, Shane McIntosh, and Andy Zaidman. Analyzing the state of static analysis: A large-scale evaluation in open source software. In 2016 IEEE 23rd International Conference on Software Analysis, Evolution, and Reengineering (SANER), volume 1, pages 470-481. IEEE, 2016.

[5] Azzedine Benameur, Nathan S Evans, and Matthew C Elder. Minestrone: Testing the soup. In CSET, 2013.

[6] Al Bessey, Ken Block, Ben Chelf, Andy Chou, Bryan Fulton, Seth Hallem, Charles Henri-Gros, Asya Kamsky, Scott McPeak, and Dawson Engler. A few billion lines of code later: using static analysis to find bugs in the real world. Communications of the ACM, 53(2):66-75, 2010.

[7] bright-tools open source project on github. C code source metrics (ccsm). https://github.com/bright-tools/ccsm

[8] CERT. Cert c coding standard (wiki). https://wiki.sei.cmu.edu/ confluence/display/c/GCC

[9] Aurelien Delaitre, Vadim Okun, and Elizabeth Fong. Of massive static analysis data. In Software Security and Reliability-Companion (SEREC), 2013 IEEE 7th International Conference on, pages 163-167. IEEE, 2013.
[10] Aurelien Delaitre, Bertrand Stivalet, Elizabeth Fong, and Vadim Okun. Evaluating bug finders-test and measurement of static code analyzers. In Complex Faults and Failures in Large Software Systems (COUFLESS), 2015 IEEE/ACM 1st International Workshop on, pages 14-20. IEEE, 2015.

[11] Lori Flynn, Ebonie McNeil, Matt Sisk, and William Snavely. Open dataset RC_data for classifier research. https://wiki.sei.cmu.edu/confluence/display/seccode/Open+Dataset+ RC Data+for+Classifier+Research August 2020.

[12] NSA Center for Assured Software. Juliet test suite c/c++ v1.2 user guide. https://samate.nist.gov/SARD/resources/Juliet_Test_Suite_v1.2_ for_C_Cpp_-_User_Guide.pdf

[13] CERT Secure Coding group. Sei cert coding standards (wiki). https: //wiki.sei.cmu.edu/confluence/display/seccode 2018. Accessed March 9, 2018.

[14] Sarah Heckman and Laurie Williams. A systematic literature review of actionable alert identification techniques for automated static code analysis. Information and Software Technology, 53(4):363-387, 2011.

[15] Intelligence Advanced Research Projects Activity (IARPA). Iarpa stonesoup. https://samate.nist.gov/SRD/view.php?tsID=102

[16] Xiaoyuan Jing, Fei Wu, Xiwei Dong, Fumin Qi, and Baowen Xu. Heterogeneous cross-company defect prediction by unified metric representation and cca-based transfer learning. In Proceedings of the 2015 10th Joint Meeting on Foundations of Software Engineering, pages 496507. ACM, 2015.

[17] kaggle. Kaggle website. https://www.kaggle.com/ 2018. Accessed March 19, 2018.

[18] Ted Kremenek, Ken Ashcraft, Junfeng Yang, and Dawson Engler. Correlation exploitation in error ranking. In ACM SIGSOFT Software Engineering Notes, volume 29, pages 83-93. ACM, 2004.

[19] Ted Kremenek and Dawson Engler. Z-ranking: Using statistical analysis to counter the impact of static analysis approximations. In International Static Analysis Symposium, pages 295-315. Springer, 2003.

[20] Gregory Larsen, EK Fong, David A Wheeler, and Rama S Moorthy. State-of-the-art resources (soar) for software vulnerability detection, test, and evaluation. Technical report, INSTITUTE FOR DEFENSE ANALYSES ALEXANDRIA VA, 2014.

[21] MITRE. Common weakness enumeration: A community-developed dictionary of software weakness types. https://cwe.mitre.org Accessed June 22, 2016.

[22] MITRE. Cwe-compatible products and services. https://cwe.mitre.org/ compatible/compatible.html 2018. Accessed March 9, 2018.

[23] Jaechang Nam and Sunghun Kim. Clami: Defect prediction on unlabeled datasets (t). In Automated Software Engineering (ASE), 2015 30th IEEE/ACM International Conference on, pages 452-463. IEEE, 2015.

[24] National Institute of Standards and Technology (NIST). Sard test suites. https://samate.nist.gov/SRD/testsuite.php

[25] Daniel Plakosh, Robert Seacord, Robert W Stoddard, David Svoboda, and David Zubrow. Improving the automated detection and analysis of secure coding violations. 2014.

[26] The GNU Project. Gnu compiler collection (gcc). https://gcc.gnu.org/

[27] Joseph R Ruthruff, John Penix, J David Morgenthaler, Sebastian Elbaum, and Gregg Rothermel. Predicting accurate and actionable static analysis warnings: an experimental approach. In Proceedings of the 30th international conference on Software engineering, pages 341-350. ACM, 2008.

[28] David Svoboda, Lori Flynn, and William Snavely. Static analysis alert audits: Formal lexicon and rules. In Proceedings of the IEEE Cybersecurity Development (SecDev) 2016. ieee, 2016.

[29] CERT Secure Coding Team. Cert rosecheckers static analysis tool for c code. http://rosecheckers.sourceforge.net

[30] Cppcheck Team. cppcheck static source code analysis tool for $\mathrm{c}$ and c++ code. https://sourceforge.net/projects/cppcheck/ 2021.

[31] Terry Yin. Lizard. https://github.com/terryyin/lizard Accessed January 29, 2018.

[32] Feng Zhang, Quan Zheng, Ying Zou, and Ahmed E Hassan. Crossproject defect prediction using a connectivity-based unsupervised classifier. In Proceedings of the 38th International Conference on Software Engineering, pages 309-320. ACM, 2016. 


\begin{tabular}{|c|c|c|c|c|c|}
\hline CWE-ID & test count & TP rate & precision & recall & accuracy \\
\hline 824 & 3239 & 0.09 & 1.00 & 1.00 & 1.00 \\
\hline 457 & 2906 & 0.06 & 1.00 & 1.00 & 1.00 \\
\hline 681 & 2871 & 0.25 & 0.85 & 0.87 & 0.93 \\
\hline 665 & 2467 & 0.03 & 1.00 & 1.00 & 1.00 \\
\hline 908 & 2451 & 0.03 & 1.00 & 1.00 & 1.00 \\
\hline 758 & 2317 & 0.00 & 1.00 & 1.00 & 1.00 \\
\hline 195 & 1485 & 0.22 & 0.73 & 0.95 & 0.91 \\
\hline 194 & 1286 & 0.25 & 0.72 & 0.95 & 0.89 \\
\hline 196 & 1265 & 0.24 & 0.83 & 0.80 & 0.92 \\
\hline 676 & 994 & 0.44 & 0.82 & 0.82 & 0.84 \\
\hline 426 & 982 & 0.43 & 0.84 & 0.74 & 0.83 \\
\hline 78 & 982 & 0.43 & 0.82 & 0.80 & 0.83 \\
\hline 704 & 866 & 0.40 & 0.82 & 0.90 & 0.88 \\
\hline 253 & 853 & 0.27 & 1.00 & 1.00 & 1.00 \\
\hline 762 & 850 & 0.67 & 1.00 & 1.00 & 1.00 \\
\hline 404 & 734 & 0.56 & 1.00 & 0.99 & 1.00 \\
\hline 761 & 698 & 0.60 & 1.00 & 1.00 & 1.00 \\
\hline 197 & 693 & 0.44 & 0.94 & 0.73 & 0.86 \\
\hline 401 & 663 & 0.53 & 1.00 & 1.00 & 1.00 \\
\hline 680 & 618 & 0.17 & 0.73 & 0.93 & 0.93 \\
\hline 476 & 513 & 0.23 & 0.99 & 0.99 & 1.00 \\
\hline 690 & 501 & 0.17 & 0.99 & 0.99 & 1.00 \\
\hline 190 & 461 & 0.16 & 1.00 & 1.00 & 1.00 \\
\hline 20 & 446 & 0.64 & 1.00 & 1.00 & 1.00 \\
\hline 188 & 424 & 0.33 & 0.83 & 0.91 & 0.91 \\
\hline 672 & 417 & 0.60 & 1.00 & 1.00 & 1.00 \\
\hline 134 & 381 & 0.44 & 0.97 & 0.79 & 0.90 \\
\hline 775 & 376 & 0.18 & 0.98 & 0.97 & 0.99 \\
\hline 191 & 361 & 0.29 & 0.77 & 0.79 & 0.87 \\
\hline 129 & 336 & 0.49 & 1.00 & 0.98 & 0.99 \\
\hline 590 & 300 & 1.00 & 1.00 & 1.00 & 1.00 \\
\hline 628 & 279 & 0.88 & 0.98 & 0.98 & 0.97 \\
\hline 369 & 234 & 0.47 & 0.96 & 0.97 & 0.97 \\
\hline 606 & 232 & 0.31 & 1.00 & 1.00 & 1.00 \\
\hline 119 & 225 & 0.73 & 1.00 & 1.00 & 1.00 \\
\hline 252 & 167 & 0.74 & 1.00 & 1.00 & 1.00 \\
\hline 456 & 148 & 0.54 & 1.00 & 1.00 & 1.00 \\
\hline 909 & 139 & 0.49 & 1.00 & 1.00 & 1.00 \\
\hline 125 & 135 & 0.56 & 1.00 & 1.00 & 1.00 \\
\hline 121 & 118 & 0.82 & 0.93 & 0.95 & 0.90 \\
\hline 122 & 116 & 0.83 & 0.98 & 0.90 & 0.90 \\
\hline 843 & 115 & 0.23 & 0.81 & 0.78 & 0.90 \\
\hline 126 & 71 & 0.83 & 0.93 & 0.97 & 0.92 \\
\hline 563 & 65 & 0.17 & 1.00 & 1.00 & 1.00 \\
\hline 377 & 64 & 0.00 & & & 1.00 \\
\hline 415 & 37 & 0.73 & 1.00 & 0.96 & 0.97 \\
\hline 468 & 36 & 0.36 & 1.00 & 1.00 & 1.00 \\
\hline 469 & 35 & 0.37 & 1.00 & 1.00 & 1.00 \\
\hline 398 & 34 & 0.38 & 1.00 & 1.00 & 1.00 \\
\hline 192 & 33 & 0.00 & & & 1.00 \\
\hline 480 & 32 & 0.97 & 1.00 & 1.00 & 1.00 \\
\hline 783 & 32 & 0.97 & 1.00 & 1.00 & 1.00 \\
\hline 127 & 27 & 0.96 & 0.96 & 0.96 & 0.93 \\
\hline 327 & 25 & 1.00 & 1.00 & 1.00 & 1.00 \\
\hline 416 & 23 & 0.56 & 1.00 & 1.00 & 1.00 \\
\hline 688 & 22 & 0.00 & & & 1.00 \\
\hline 569 & 20 & 0.95 & 1.00 & 1.00 & 1.00 \\
\hline 787 & 20 & 0.60 & 1.00 & 1.00 & 1.00 \\
\hline 120 & 19 & 1.00 & 1.00 & 1.00 & 1.00 \\
\hline 131 & 19 & 1.00 & 1.00 & 1.00 & 1.00 \\
\hline 170 & 19 & 1.00 & 1.00 & 1.00 & 1.00 \\
\hline 328 & 19 & 1.00 & 1.00 & 1.00 & 1.00 \\
\hline 467 & 19 & 1.00 & 1.00 & 1.00 & 1.00 \\
\hline 587 & 18 & 0.39 & 1.00 & 1.00 & 1.00 \\
\hline 570 & 16 & 1.00 & 1.00 & 1.00 & 1.00 \\
\hline 367 & 13 & 1.00 & 1.00 & 1.00 & 1.00 \\
\hline 465 & 13 & 1.00 & 1.00 & 1.00 & 1.00 \\
\hline 597 & 13 & 1.00 & 1.00 & 1.00 & 1.00 \\
\hline 483 & 11 & 0.00 & & & 1.00 \\
\hline 338 & 7 & 1.00 & 1.00 & 1.00 & 1.00 \\
\hline 478 & 7 & 1.00 & 1.00 & 1.00 & 1.00 \\
\hline 482 & 7 & 1.00 & 1.00 & 1.00 & 1.00 \\
\hline 484 & 7 & 1.00 & 1.00 & 1.00 & 1.00 \\
\hline 123 & 6 & 0.83 & 0.83 & 1.00 & 0.83 \\
\hline 561 & 5 & 1.00 & 1.00 & 1.00 & 1.00 \\
\hline 571 & 4 & 1.00 & 1.00 & 1.00 & 1.00 \\
\hline 466 & 1 & 1.00 & 1.00 & 1.00 & 1.00 \\
\hline 562 & 1 & 1.00 & & 0.00 & 0.00 \\
\hline 835 & 1 & 1.00 & 1.00 & 1.00 & 1.00 \\
\hline
\end{tabular}

\begin{tabular}{llrrrr}
\hline CERT rule & test count & TP rate & precision & recall & accuracy \\
\hline ARR30-C & 2123 & 0.30 & 0.81 & 0.84 & 0.89 \\
ARR36-C & 2015 & 0.06 & 1.00 & 1.00 & 1.00 \\
ARR38-C & 973 & 0.16 & 0.84 & 0.85 & 0.95 \\
ARR39-C & 631 & 0.03 & 1.00 & 0.94 & 1.00 \\
CON43-C & 615 & 0.12 & 0.81 & 0.85 & 0.96 \\
DCL30-C & 372 & 0.42 & 0.97 & 0.78 & 0.90 \\
ENV33-C & 371 & 0.16 & 0.98 & 0.97 & 0.99 \\
ERR33-C & 271 & 0.07 & 1.00 & 1.00 & 1.00 \\
ERR34-C & 259 & 1.00 & 1.00 & 1.00 & 1.00 \\
EXP33-C & 240 & 0.00 & & & 1.00 \\
EXP34-C & 234 & 0.47 & 0.96 & 0.97 & 0.97 \\
EXP37-C & 197 & 1.00 & 1.00 & 1.00 & 1.00 \\
EXP45-C & 186 & 0.40 & 0.81 & 0.95 & 0.89 \\
EXP46-C & 140 & 0.12 & 1.00 & 1.00 & 1.00 \\
FIO30-C & 106 & 0.85 & 0.97 & 0.92 & 0.91 \\
FIO42-C & 93 & 1.00 & 1.00 & 1.00 & 1.00 \\
FIO47-C & 57 & 0.75 & 0.93 & 0.93 & 0.89 \\
FLP32-C & 48 & 0.31 & 0.93 & 0.93 & 0.96 \\
FLP34-C & 35 & 0.37 & 1.00 & 1.00 & 1.00 \\
INT30-C & 31 & 0.26 & 1.00 & 1.00 & 1.00 \\
INT31-C & 18 & 0.39 & 1.00 & 1.00 & 1.00 \\
INT32-C & 13 & 1.00 & 1.00 & 1.00 & 1.00 \\
INT33-C & 7 & 1.00 & 1.00 & 1.00 & 1.00 \\
INT36-C & 1 & 1.00 & & 0.00 & 0.00 \\
MEM30-C & 1 & 1.00 & 1.00 & 1.00 & 1.00 \\
\hline & \multicolumn{3}{c}{ TABLE V } & & \\
PER & & &
\end{tabular}

PERFORMANCE OF LIGHTGBM ON TEST SETS COMPOSED OF FUSED ALERTS THAT CORRESPOND TO SPECIFIC CERT RULES.

\begin{tabular}{lrr}
\hline Rank & Feature & Importance \\
\hline 1 & CCSM FUNC_CALLED_BY_LOCAL & 0.423 \\
2 & checkerID & 0.294 \\
3 & CCSM RAW_KW_LONG_CNT & 0.047 \\
4 & Tool_X Metric 1 & 0.031 \\
5 & Tool_X Metric 2 & 0.026 \\
6 & CWE & 0.025 \\
7 & Lizard Parameters & 0.015 \\
8 & Tool_X Metric 3 & 0.012 \\
9 & Lizard Number_of_Tokens & 0.008 \\
10 & Tool_X Metric 4 & 0.006 \\
\hline
\end{tabular}

FEATURE IMPORTANCE IN LightGBM: TOP 10.

PERFORMANCE OF LightGBM ON TEST SETS COMPOSED OF META ALERTS FOR SPECIFIC CWE IDS. 